\title{
Study of SVM algorithm for Data Mining in Big Data
}

\author{
V.Nanda Kumar ${ }^{1 *}$, Vinoth N.A.S. ${ }^{2}$, Mohamed Sanjarkhan ${ }^{3}$ \\ ${ }^{1}$ Assistant Professor, Department of Computer Science and Engineering, PSNA College of Engineering and Technology, \\ Dindigul, Tamil Nadu. \\ ${ }^{2}$ Assistant Professor, SRM Institute of Science and Technology, \\ ${ }^{3}$ Assistant professor,Sri Sairam institute of technology
}

\begin{abstract}
Data mining is a process which finds useful patterns from large amount of data.These days, there are an excessive number of Data Mining Algorithms present. Support Vector Machine (SVM) is assuming a crucial function as it gives strategies so as to acquire brings about a viable route and with an elevated level of value. In this paper, we examine about the function of SVM calculation in large information from information mining viewpoint undertakings like order, bunching, expectation, estimating and others applications. In current situation world is comprised of "huge information". The principle point of this paper is to unmistakably comprehend the premise of SVM procedures in different zones. In our perspective, we have assessed the quantity of exploration distributions that have been advanced in various rumored diaries for the information mining applications and furthermore recommended a potential number of issues of SVM.
\end{abstract}

Keywords

Support Vector Machine (SVM), Data Mining, Artificial Neural Network (ANN)

Article Received: 20 September 2020, Revised: 30 November 2020, Accepted: 18 December 2020

\section{Introduction}

Support Vector Machines presented in COLT-92 by Boseretal. (1992). Tsukimoto (2000) built up a calculation for removing rules from prepared neural organizations, the calculation can be utilized for any neural organization and the yield work was droning, for example, sigmoid capacity.

SVM calculation utilized for characterization, relapse, and inconsistency recognition. It likewise gives the adaptability and ease of use that are required for creating the nature of information in information mining framework. This paper presents and investigations SVM calculation, which will help to new analysts to comprehend the tuning, diagnostics and information readiness cycle and points of interest of SVM in Data Mining. SVM can be material for complex genuine issues, for example, many example acknowledgment and relapse assessment issues and to the issues of reliance assessment, determining and developing canny machines

SVM keeps up its notoriety in the most recent decade. It likewise sent to different areas of uses. SVM helps for learning arrangement, relapse or positioning capacity. SVM relies upon Structural Risk Minimization principle(SRM) i.e., the calculation depends on ensured hazard limits of measurable learning hypothesis. Because of this rule, SVM can catch highlight spaces. It likewise centers to decide the area of choice limits. It is otherwise called a hyperplane. That gives the ideal division of classes. Therefore, it makes the greatest separation between the hyperplane.

The productivity of SVM not relies upon the element of grouped substances. Nonetheless, SVM is the most vigorous and exact order procedure. In addition, there are a few issues. The information investigation in SVM relies upon raised quadratic programming.

In MLP classifiers, the qualities are refreshed during the preparation stage, in association with this, the choice limits between classes obtained via preparing are aberrant to fearless and the speculation capacity is reliant on the preparation approach. In logical inconsistency to this, in SVM the choice limits are legitimately decided from the preparation informational index. In view of this, we can augment the limits of isolating edges in include space.

SVM can likewise stretch out to learn non-direct choice capacities. At first it extends the info information onto a high-dimensional space. As by utilizing portion works and figuring a straight arrangement issue. The subsequent element space is a lot bigger than the size of a dataset. Along these lines it is absurd to expect to store on famous PCs.

Investigating this issues prompts a few decay based calculations. The fundamental thought of disintegration technique is to separate the factors into two sections:

A lot of free factors called as a working set. It very well may be refreshed in every emphasis and set of fixed factors. Those are fixed during a specific execution. Presently, this methodology needs to rehash until the end conditions are fulfilled.

The SVM was created for parallel order. Additionally, it isn't so natural to broaden it for multi-class arrangement issue. The essential plan to apply multi-characterization to 
SVM is to decay the multi-class issues into a few two-class issues. That can be tended to utilizing a few SVMs.

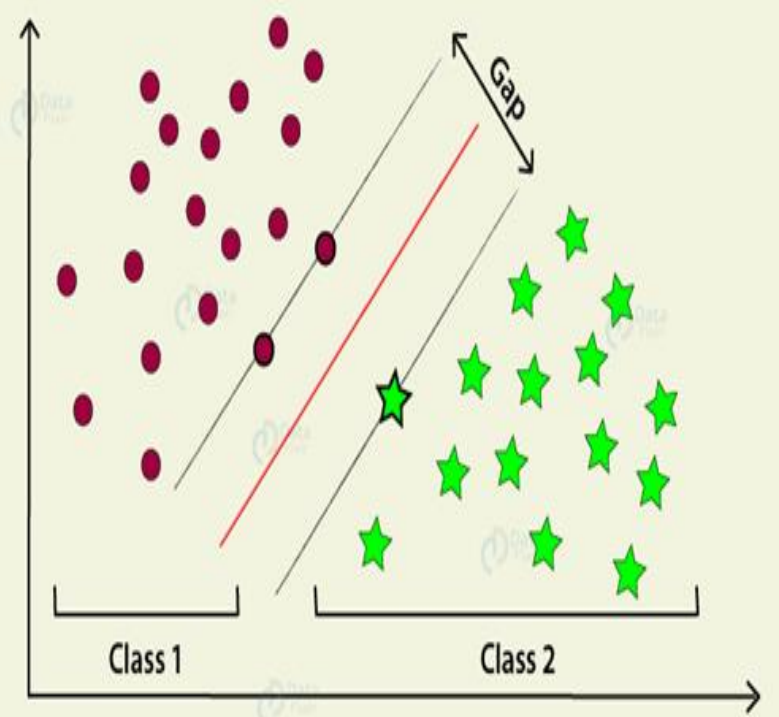

Figure 1.Linearly Separable Samples Indicated in a

Hyperplane

There can be numerous lines/choice limits to isolate the classes in n-dimensional Space, yet we have to discover the best choice limit that assists with arranging the information focuses. This best limit is known as the hyperplane of SVM. The elements of the hyperplane rely upon the highlights present in the dataset, which implies on the off chance that there are 2 highlights (as appeared in picture), at that point hyperplane will be a straight line. Furthermore, in the event that there are 3 highlights, at that point hyperplane will be a 2-measurement plane. We generally make a hyperplane that has a most extreme edge, which implies the greatest separation between the information focuses.

\section{Support Vectors}

The information focuses or vectors that are the nearest to the hyperplane and which influence the situation of the hyperplane are named as Support Vector. Since these vectors uphold the hyperplane, thus called a Support vector.

SVM goes under the sort of managed AI calculation that gives an investigation of information for characterization and relapse examination. During a similar time, they can be utilized for relapse, SVM is generally utilized for order. We do plotting in the $n$-dimensional space. Estimation of each component is same as the estimation of the particular arrange. At that point, we discovering ideal hyperplanehas the effect between the two classes.

These help vectors are the facilitate portrayals of explicit perception. It is an outskirts technique for isolating the two classes.

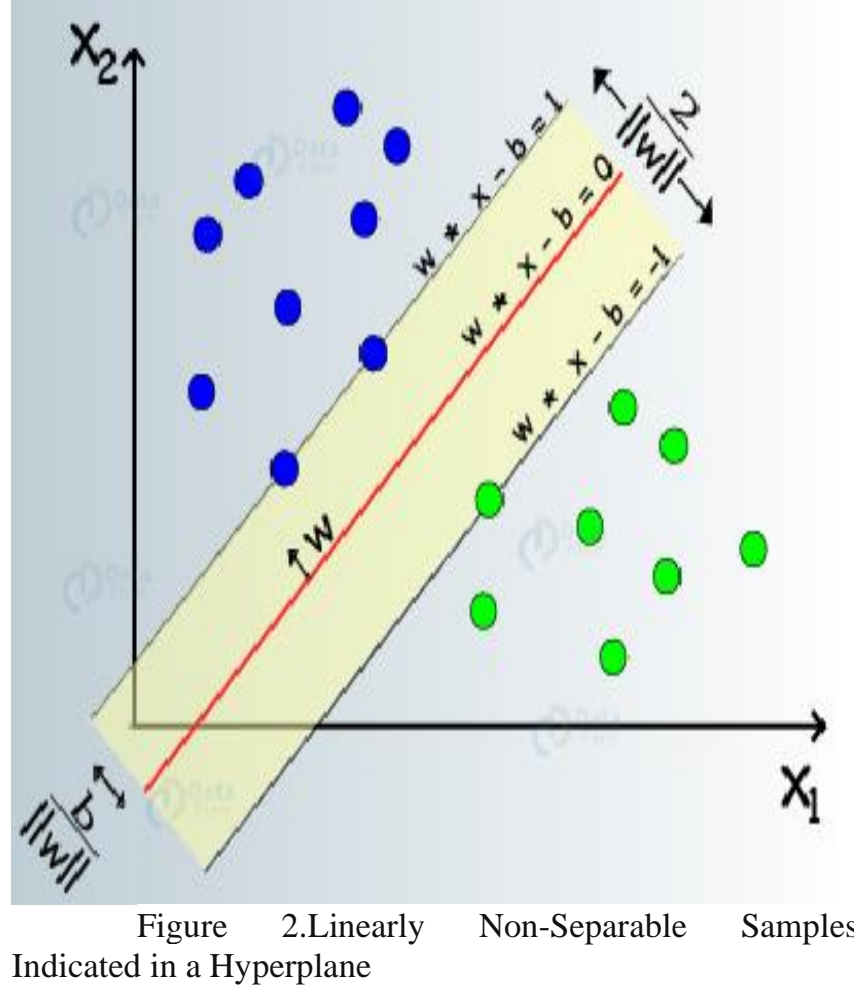

The essential rule behind the working of Support vector machines is direct - Create a hyperplane that separates the dataset into classes. Let us start with an example issue. Assume, for a given dataset, you need to classify red triangles from blue circles. Your point is to make a line that characterizes the information into two classes, making a contrast between red triangles and blue circles.

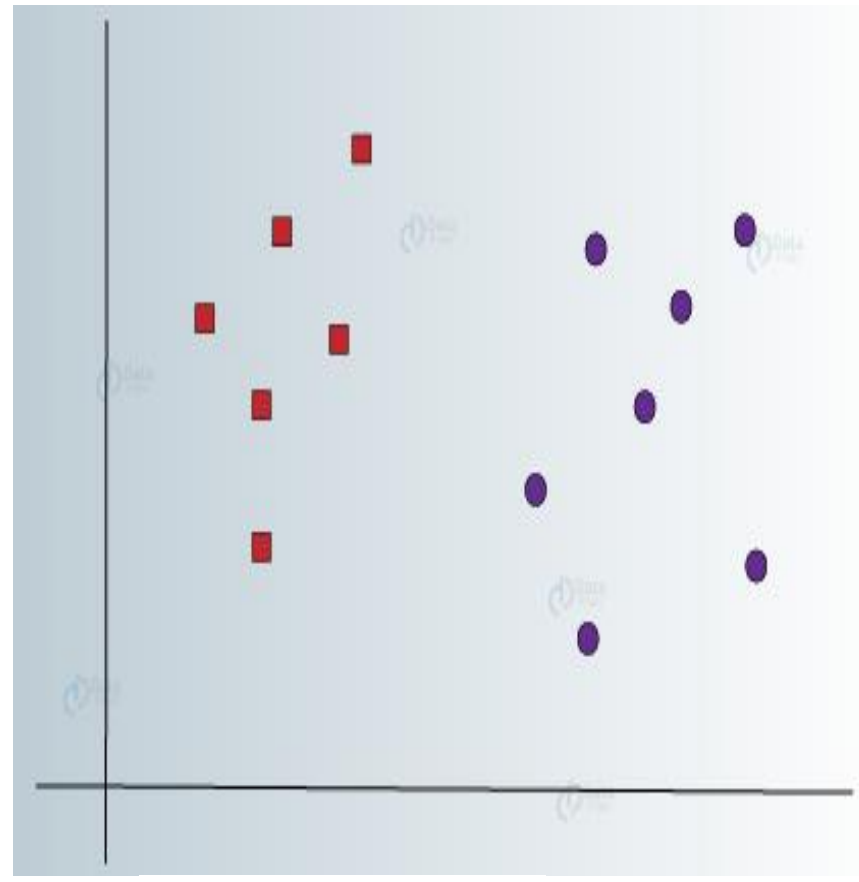

Figure 3. Dataset into classes 
At some time one can envision a reasonable line that isolates the two classes, there can be numerous lines can capable carry out this responsibility. Accordingly, there is certifiably not a solitary line that you can concur on which we can play out this errand. Let us consider a portion of the lines that can have any kind of effect between the two classes as follows -

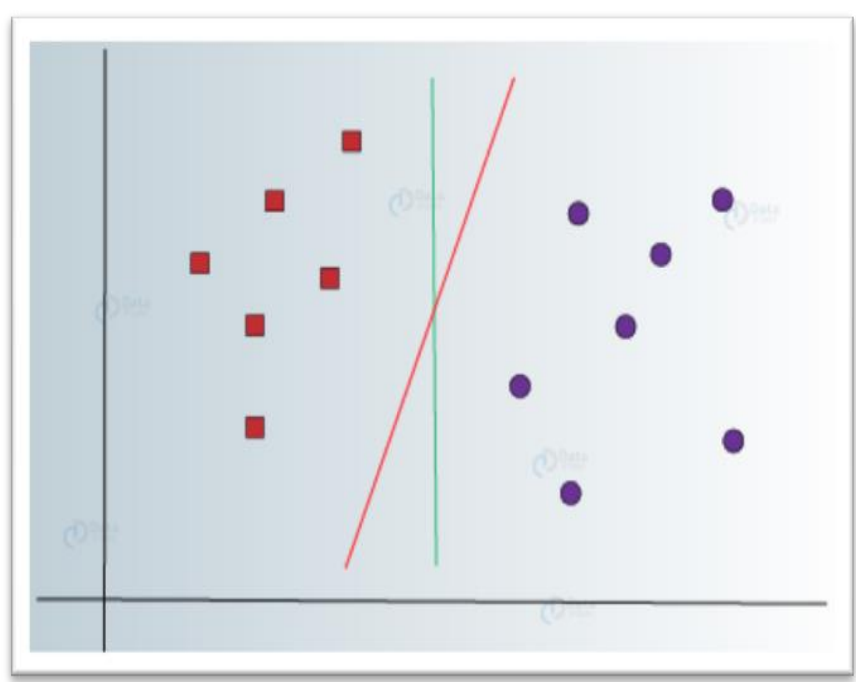

Figure 4. Clear partition between two classes

In the above picture, we have a green line and a red line. Which one improve separate the information into two classes? In the event that you pick the red line, at that point it is the ideal line that segments the two classes appropriately. Nonetheless, we actually have not represented the way that it is the widespread line that would group our information most effectively.

Fake Neural Network utilized for Association Rules, Clustering, Prediction, and Classification. ANN consolidates with different calculations so as to discover the high exactness of information as contrast with conventional calculation. The part of ANN assumes a significant function in estimating or expectation about games and climate. This produces high exact expectations than that of conventional calculation. The authoritative of neural organization model and information mining strategy can expand the proficiency of information mining strategies.

The green line can't be the ideal line as it lies excessively near the red class. Hence, it doesn't give a legitimate speculation, so we need to think about this as an our ultimate objective.

As controlled by SVM, we should discover the focuses that lie closer to both the classes. These focuses are known as help vectors. By following this, we need to discover the closeness between our isolating plane and the help vectors. The separation between the focuses and the isolating line is known as edge. The goal of a SVM calculation is to amplify this edge. At the point when the edge arrives at its most extreme, at the same time the hyperplane turns into the ideal one.

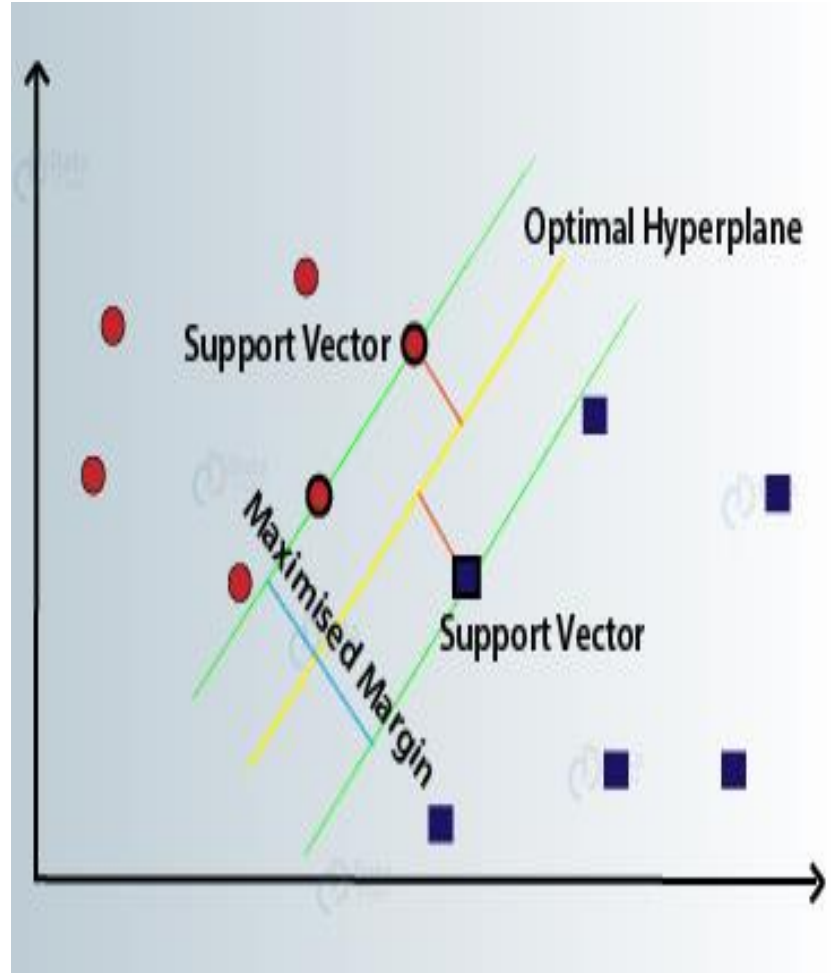

Figure 5. Proximity between dividing plane and support vectors

The SVM model endeavors to expand the separation between the two classes by setting up an all around characterized choice limit. In the above certainty, our hyperplane isolated the information. Despite the fact that our information was in two measurements, the hyperplane was in a single measurement. For higher measurements, express a n-dimensional Euclidean Space, we have a n-1 dimensional subset that separates the space into two detached segments.

Table 1. Pseudo code for SVM

Require: A linear separable set $\mathrm{S}$, learning rate $\square \square \square \square^{\square}$
$\mathrm{u}_{0} \square \square \square \square \square \mathrm{v} \square \square \square \square \square \square \mathrm{t} \square \square \square \square$
$\mathrm{R}=$ max $\left\|\mathrm{x}_{\mathrm{i}}\right\| 1 \square \square \mathrm{i} \square \square \mathrm{1}$
while at least one mistake is made in the for loop do
for $\mathrm{i} \square \square 1_{1}, \ldots, 1$ do
if $\mathrm{y}_{\mathrm{i}}\left(\square \square \mathrm{u}_{\square \square}, \mathrm{x}_{\mathrm{i}} \square \square \square \square \square \mathrm{v}_{\mathrm{t}}\right) \square \square 0$ then
$\mathrm{u}_{\mathrm{t}+1} \square \square \mathrm{u}_{\mathrm{t}} \square \square \mathrm{x}_{\mathrm{i}}$
$\mathrm{v}_{\mathrm{k}} \square \square \square \mathrm{y}_{\mathrm{i}} \square \square^{\square} \square\left(\right.$ updating bias ${ }^{1}$ )
$\mathrm{t} \square \square \mathrm{t} \square 1$
end if
end for
end while
Return $\mathrm{u}_{\mathrm{t}}, \mathrm{v}_{\mathrm{t}}$, where tis the number of mistakes




\section{Applications of SVM}

Face Detection:

So as to decide the face, it classifies the pieces of the picture as face and non-face. It contains preparing information of $n$ $\mathrm{x} n$ pixels with two-class esteems +1 for face and -1 for non-face. After that it separates data from every pixel as face or non-face. In light of the pixel splendor it makes a square limit around faces and arranges each picture by utilizing a similar cycle.

Text and Hypertext Categorization:

SVM permits text and hypertext order for the two sorts of models inductive and transductive. With the assistance of preparing information, SVM can ready to characterize records into various classifications, for example, magazine, messages, and pages.

Models:

- Classification of magazine into "business" and "Motion pictures"

- Classification of website pages into individual home pages and others

For each report, score is determined and look at it with a predefined edge esteem. At the point when the record score outperforms edge esteem, at that point the archive is ordered into an unmistakable class. Something else, think about it as an overall report. New occasions could be characterized dependent on processing score for each record and contrasting it and the educated edge.

\section{Grouping of Images}

SVMs can group pictures prompts achieve preferable higher hunt exactness over conventional inquiry based refinement plans

\section{Bioinformatics}

In the territory of computational science, the basic issue is protein distant homology recognition. SVM is the best strategy to explain this issue. In most recent couple of years, SVM calculations have been incredible in degree applied for protein distant homology location. In addition, these calculations can likewise be incredibly utilized for recognizing among organic successions. For instance, gathering of qualities, patients dependent on their qualities, and numerous other organic issues.

Penmanship Recognition

SVM can likewise be utilized to distinguish transcribed characters that help for information passage and approving marks on records.

\section{Geo and Environmental Sciences}

SVM is appropriate for geo (spatial) and spatiotemporal natural information investigation and demonstrating arrangement.

\section{Summed up Predictive Control}

SVM-based GPC can be utilized to control disordered elements alongside helpful boundaries. It gives colossal execution in controlling the frameworks. It follows disorderly elements concerning the nearby adjustment of the objective.

Utilizing SVMs for controlling disorderly frameworks has the accompanying points of interest-

- Relatively little boundary calculations can be utilized to divert a tumultuous framework to the objective.

- This framework can decrease hanging tight an ideal opportunity for turbulent frameworks.

- Maintains the exhibition of frameworks.

\section{Penmanship Recognition}

The arrangement of utilizing feed-forward organizations is clear way to deal with perceive manually written characters. The bitmap example of the manually written character is contribution, with the right letter or digit as the ideal yield. Such modules need the clients need to prepare the organization by giving the program with their transcribed examples.

Feed-forward organizations have the accompanying qualities:

a. At first, they request perceptrons in layers, the main layer utilized for taking sources of info and the last layer for delivering yields. The center layers are shrouded layer, since it had no association with the outside world. 
b. Each perceptron in one layer is connected to each other perceptron on the following layer. Consequently data is "feed forward" starting with one layer then onto the next in an unaltered way. This shows that why we call these organizations feed-forward organizations.

c. There is no association among perceptrons in a similar layer.

The two normal uses of penmanship acknowledgment are:

- Optical character acknowledgment for information section

- Validation of marks on a bank check

Mobile Salesman Problem

The mobile sales reps issue assists with finding the most limited conceivable way to go among all urban areas in a given territory. Neural Networks can be a superior answer for take care of this issue.

A neural organization calculation, for example, a hereditary calculation starts with arbitrary direction of the organization, to tackle the issue. This calculation picks a city in an arbitrary way each time and finds the closest city. Hence, these cycles prop up a few times. After each cycle, the state of the organization changes and organization meets to a ring around all the urban communities. This calculation can ready to limit the length of rings. By along these lines, we can assess the voyaging issue.

\section{Image Compression}

A Neural Network utilized for picture pressure contains the equivalent size of information and yield layer. The halfway layer is of littler size. The proportion of the information layer to the middle of the road layer is the pressure proportion of the organization.

We can get the correlation proportion for picture pressure utilizing the accompanying recipe:

\section{Correlation Ratio $=$ Input Layer/Intermediate Layer}

Rationale of information pressure neural organizations is to store, scramble and re-make the real picture once more.

Stock Exchange Prediction
To make a financial exchange expectation in better exactness, neural organizations is most ideal approach to deal with. For complex business organizations, making forecasts for stock trade is normal. Expectations should be possible by utilizing boundaries, for example, current patterns, political circumstance, general visibility, and financial analyst's recommendation.

Additionally neural organizations can likewise be utilized in money forecast, business disappointment expectation, obligation hazard evaluation, and credit endorsement.

Organizations, for example, Futures guarantee astounding $198.5 \%$ returns over a 3-year time frame utilizing their neural organization expectation techniques.

\section{Limitations}

As the prominence of SVM is expanding step by step, so unique examination applications depending on SVM must be distributed, to encourage the wide widen extent of SVM, in the scholarly and commonsense fields. Nonetheless, not many analysts have pointed constraints of SVM on which work must be completed like: (1) The choice of bit for an issue (2) The useful speed of the machine in preparing and testing, (3) Slower Convergence rate at testing phase,(4) Choosing great quality bit boundaries, (5) Large necessities of memory space to actualize the model (6) Choosing either parametric or non-parametric strategy for usage. This coordination of strategies and cross-disciplinary examination may advance new experiences for critical thinking with SVM.

Inconvenience of neural organizations is their "discovery" nature. For instance, when you put a picture of a feline into a neural organization and it predicts it to be a vehicle, it is difficult to comprehend what made it show up at this forecast. At the point when you have highlights that are human interpretable, it is a lot more clear the reason for the misstep. By examination, calculations like choice trees are truly interpretable. This is significant in light of the fact that in certain spaces, interpretability is basic.

\section{Conclusion}

Hence, we can presume that the SVMs can cause the dependable forecast as well as to can decrease excess data. The outcomes acquired by SVM can likewise be equivalent with the outcomes got by different methodologies.

Backing Vector Machine is a quickly expanding field with guarantee for more noteworthy materialness in all space of exploration. A few exercises like Data cleaning, Data Transformation and Outlier recognition are markable issue for an informational collections since a portion of the characteristics esteems can't be gotten as a rule. So treatment 
of missing qualities for anticipating issue is a difficult undertaking.

Unpredictability of dealing with the enormous dataset for any application is a typical issue since a large portion of characterization calculations are not appropriate to deal with it.

Correlations of Support Vector Machine (SVM) and Artificial Neural Network (ANN) calculations are done dependent on the presentation factors characterization precision and execution time. From the examination, it tends to be presumed that the ANN accomplishes expanded order execution, yields results that are precise, thus it is considered as best classifier when contrasted and SVM classifier calculation. Practically, SVM classifier characterizes the information with least execution time. In future, ANN calculation is upgraded to limit the execution time.

Despite the fact that there are different procedures exist, still there is a need of best way to deal with settle the accompanying exploration issues.

- Number of preparing information focuses in the Learning period of SVM scale.

- Increasing informational collection size, prompts hinder the cycle in the learning stage.

- A barely any exercises like Data cleaning, Data Transformation and Outlier location are noteworthy issue in an informational collections since a portion of the property estimations can't be gotten normally. Henceforth, treatment of missing qualities utilized for characterization or determining issue is a difficult assignment.

- Most of the arrangement calculations are not appropriate for taking care of multifaceted nature of huge dataset in any application is an ongoing issue.

- $\quad$ Selecting most proper example of information for arrangement rather than the flawless information is another danger for showing signs of improvement result.

Choosing the appropriate characterization strategies absent a lot of calculation intricacy is another positive bearing yet the adequacy ought not be influenced.

The ANN is the valuable model and it could be applied in critical thinking and AI. The figuring World has more to pick up from the Neural Network. In this way, capacity to learn by model makes them entirely adaptable and groundbreaking. Diverse sort of issues, ANN is the best to use, on the opposite side it is fundamental to comprehend the potential just as the restrictions of the Neural Network.

Choosing the fitting arrangement methods with no calculation multifaceted nature is another positive way however the adequacy ought not be influenced.

\section{References}

[1] Yadav, R. K., \&Sachan, A. K. Literature Review on Artificial Neural Network and Support Vector Machine anchored in Face Recognition System.

[2] Nayak, J., Naik, B., \&Behera, H. (2015). A comprehensive survey on support vector machine in data mining tasks: applications \& challenges. International Journal of Database Theory and Application, 8(1), 169-186.

[3] Gopika, S., \&Vanitha, M. (2017). Survey on Prediction of Kidney Disease by using Data Mining Techniques. International Journal of Advanced Research in Computer and Communication Engineering, 6(1).

[4] R. Sathish, R. Manikandan, S. Silvia Priscila, B. V. Sara and R. Mahaveerakannan, "A Report on the Impact of Information Technology and Social Media on Covid-19," 2020 3rd International Conference on Intelligent Sustainable Systems (ICISS), Thoothukudi, India, 2020, pp. 224-230, doi: 10.1109/ICISS49785.2020.9316046.

[5] Manikandan, $\mathrm{R}$ and Dr.R.Latha (2017). "A literature survey of existing map matching algorithm for navigation technology. International journal of engineering sciences \& research technology", 6(9), 326-331.Retrieved September $15,2017$.

[6] Chen, X. Y., Chau, K. W., \&Busari, A. O. (2015). A comparative study of population-based optimization algorithms for downstream river flow forecasting by a hybrid neural network model. Engineering Applications of Artificial Intelligence, 46, 258-268. 